\title{
Research Article \\ Comparison between Certain Equivalent Norms Regarding Some Familiar Properties Implying WFPP
}

\author{
Helga Fetter and Berta Gamboa de Buen \\ Centro de Investigación en Matemáticas (CIMAT), Apartado Postal 402, 36000 Guanajuato, GTO, Mexico \\ Correspondence should be addressed to Helga Fetter, fetter@cimat.mx \\ Received 13 December 2010; Accepted 1 March 2011 \\ Academic Editor: Enrique Llorens-Fuster \\ Copyright (C) 2011 H. Fetter and B. Gamboa de Buen. This is an open access article distributed \\ under the Creative Commons Attribution License, which permits unrestricted use, distribution, \\ and reproduction in any medium, provided the original work is properly cited. \\ In a Banach space with a basis we define a similar norm to the norm shown by Lin to make $l_{1}$ \\ into a space with FPP and make a comparative study of certain geometric properties such as the \\ Opial property, WNS, and uniform nonsquareness of the original space and the space with the new \\ norm.
}

\section{Introduction}

Dowling et al. in [1] defined a norm in $l_{1}$ which was used by Lin [2] to exhibit an equivalent norm which makes $l_{1}$ into a space with the fixed point property (FPP). A similar norm can be defined in every Banach space $X$ with a basis. Since $l_{1}$ with its usual norm does not have FPP, we asked ourselves if this norm in these spaces would also improve properties that imply the weak fixed point property (WFPP). We found out that in some instances it does, in some cases the original norm has better properties, and in some cases you cannot compare them. We give several examples to illustrate our assertions.

\section{The $\Gamma$ Norm in a Banach Space}

We start by giving the definition of the generalization of the norm used by Lin in a Banach space with a basis.

Definition 2.1. Let $(X,\|\cdot\|)$ be a Banach space with a basis $\left\{e_{n}\right\}$. Let $x=\sum_{i=1}^{\infty} x_{i} e_{i} \in X$ and $Q_{n}: X \rightarrow X$ be the projection $Q_{n}\left(\sum_{i=1}^{\infty} x_{i} e_{i}\right)=\sum_{i=n}^{\infty} x_{i} e_{i}$. The basis $\left\{e_{n}\right\}$ is called premonotone 
if $\left\|Q_{n} x\right\| \geq\left\|Q_{n+1} x\right\|$ and monotone if $\left\|P_{n} x\right\| \leq\left\|P_{n+1} x\right\|$ where $P_{n} x=\left(I-Q_{n+1}\right) x$ for every $x \in X$ and for every $n \in \mathbb{N}$.

Definition 2.2. Let $(X,\|\cdot\|)$ be a Banach space with a basis $\left\{e_{n}\right\}$ and $\Gamma=\left\{\gamma_{n}\right\} \subset \mathbb{R}$ with $0<\gamma_{n}<$ $\gamma_{n+1}$ and $\lim _{n} \gamma_{n}=1$. Let $x=\sum_{i=1}^{\infty} x_{i} e_{i} \in X$. Then if

$$
\|\| x\left\|\mid=\sup _{n} \gamma_{n}\right\| Q_{n} x \|,
$$

$|\|\cdot\||$ is a norm in $X$ which we will call $\Gamma$-norm.

Clearly

$$
r_{1}\|x\| \leq|\|x\|| \leq\left(\sup _{n}\left\|Q_{n}\right\|\right)\|x\| .
$$

Observe that, if $\left\{e_{n}\right\}$ is a basis in $(X,\|\cdot\|)$, then it is always premonotone in $(X,|\|\cdot\||)$ and, if $\left\{e_{n}\right\}$ is monotone in $(X,\|\cdot\|)$, then it is also monotone in $(X,|\|\cdot\||)$. Also observe that since for every $x \in X$ we have that $\lim _{n} \gamma_{n}\left\|Q_{n} x\right\|=0$, there exists $n_{0}$ such that $\|x\| \mid=$ $\gamma_{n_{0}}\left\|Q_{n_{0}} x\right\|$.

Next we define the properties related to wfpp we are going to analyze. The definition of GGLD is not the original one found in [3], but an equivalent one found in [4].

Definition 2.3. Let $Y$ be a Banach space.

(1) $Y$ has the Opial property if for every weakly null sequence $\left\{x_{n}\right\} \subset Y$ and for every $x \in Y, x \neq 0$,

$$
\lim \sup _{n}\left\|x_{n}\right\|<\lim \sup _{n}\left\|x_{n}-x\right\|
$$

(2) If $Y$ has a basis, it has the generalized Gossez-Lami Dozo property (GGLD) [4] if, for every weakly null normalized block basic sequence $\left\{y_{n}\right\}$, we have that

$$
\limsup _{n}\left\|y_{i}-y_{j}\right\|>1
$$

(3) A bounded sequence $\left\{y_{n}\right\} \subset Y$ is called diametral if

$$
\lim _{n} d\left(y_{n+1}, \operatorname{conv}\left\{y_{i}\right\}_{i=1}^{n}\right)=\operatorname{diam}\left\{y_{n}\right\}_{n=1}^{\infty} .
$$

$Y$ has weak normal structure (WNS) if there is no weakly null diametral nonzero sequence in $Y$.

(4) The coefficient $J(Y)$, related to uniform nonsquareness, since $J(Y)<2$ if and only if $Y$ is uniformly nonsquare, is given by

$$
J(Y)=\sup \left\{\min (\|x+y\|,\|x-y\|): x, y \in B_{Y}\right\}
$$


(5) The coefficient $R(Y)$ [5] is defined by

$$
R(Y)=\sup \left\{\liminf _{n}\left\|x_{n}+x\right\|:\{x\},\left\{x_{n}\right\} \subset B_{Y}, x_{n} \underset{w}{\longrightarrow} 0\right\} .
$$

(6) Coefficients $\operatorname{RW}(a, Y)$ and $\operatorname{MW}(Y)$ [6] are defined as follows: for each $a>0$

$$
\begin{gathered}
\operatorname{RW}(a, Y)=\sup \left\{\min \left(\liminf _{n}\left\|x_{n}+x\right\|, \underset{n}{\liminf }\left\|x_{n}-x\right\|\right):\|x\| \leq a,\left\{x_{n}\right\} \subset B_{Y}, x_{n} \underset{w}{\longrightarrow} 0\right\}, \\
\operatorname{MW}(Y)=\sup \left\{\frac{1+a}{\operatorname{RW}(a, Y)}: a>0\right\} .
\end{gathered}
$$

It is known that GGLD $\Rightarrow$ WNS $\Rightarrow$ wfpp and the Opial property implies wfpp. Also

$$
\begin{aligned}
& R(Y)<2 \Longrightarrow \operatorname{MW}(Y)>1 \Longrightarrow \mathrm{wfpp}, \\
& J(Y)<2 \Longrightarrow \operatorname{MW}(Y)>1 \Longrightarrow \mathrm{wfpp} .
\end{aligned}
$$

First we will show that the Opial property is inherited from $(X,|\|\cdot\||)$ to $(X,\|\cdot\|)$ and that $(X,\|\cdot\|)$ has GGLD if and only if $(X,|\|\cdot\||)$ has GGLD. In order to achieve this, we need the following result shown in [7].

Lemma 2.4. Let $(X,\|\cdot\|)$ be a Banach space with a premonotone basis $\left\{e_{n}\right\}$. Then

(1) if $\left\{x_{n}\right\}$ converges weakly to $x, \lim _{n}\left\|x_{n}-x\right\|=a$ if and only if $\lim _{n}\left|\left\|x_{n}-x\right\|\right|=a$,

(2) if $\left\{x_{n}\right\}$ converges weakly to 0 and $\lim _{n} \lim _{r}\left\|x_{n}-x_{r}\right\|=a$, there exists a subsequence $\left\{y_{n}\right\}$ of $\left\{x_{n}\right\}$ such that $\lim _{n} \lim _{r}\left|\left\|y_{n}-y_{r}\right\|\right|=a$.

Lemma 2.5. Let $(X,\|\cdot\|)$ be a Banach space with a premonotone basis $\left\{e_{n}\right\}$. If $(X,|\|\cdot\||)$ has the Opial property, then $(X,\|\cdot\|)$ also has the Opial property, but the converse is false.

Proof. Let $\left\{x_{n}\right\}$ be weakly null in $X$ and $x \in X, x \neq 0$. Then, by Lemma 2.4 and by (2.2),

$$
\lim \sup _{n}\left\|x_{n}\right\|=\lim \sup _{n}\left|\left\|x_{n}\right\|\right|<\lim \sup _{n}\left|\left\|x_{n}-x\right\|\right| \leq \lim \sup _{n}\left\|x_{n}-x\right\| .
$$

It is known that, for $1<p<\infty,\left(l_{p},\|\cdot\|\right)$ has the Opial property. Consider any $\Gamma$-norm $|\|\cdot\||$ in $l_{p}$ with the canonical basis $\left\{e_{n}\right\}$, and let $\delta>0$ be such that $\delta<\left(\left(\gamma_{2}^{p}-\gamma_{1}^{p}\right) / \gamma_{1}^{p}\right)^{1 / p}$. Then, for $n \geq 2$,

$$
\begin{gathered}
\left|\left\|\delta e_{1}+e_{n} \mid\right\|=\max \left[\gamma_{1}\left(\delta^{p}+1\right)^{1 / p}, \gamma_{n}\right]=\gamma_{n},\right. \\
\lim _{n}\left|\left\|e_{n}\right\|\right|=\lim _{n} \gamma_{n}=\lim _{n}\left|\left\|\delta e_{1}+e_{n}\right\|\right|=1 .
\end{gathered}
$$

Thus, $\left(l_{p},|\|\cdot\||\right)$ does not have the Opial property. 
Lemma 2.6. Let $(X,\|\cdot\|)$ be a Banach space with a premonotone basis $\left\{e_{n}\right\}$. Then, $(X,\|\cdot\|)$ has GGLD if and only if $(X,|\|\cdot\||)$ has $G G L D$.

Proof. Let $\left\{y_{n}\right\}$ be a weakly null normalized block basic sequence. By Lemma 2.4, $\lim _{n}\left\|y_{n}\right\|$ exists if and only if $\lim _{n}\left|\left\|y_{n}\right\|\right|$ exists and in this case $\lim _{n}\left\|y_{n}\right\|=\lim _{n}\left|\left\|y_{n}\right\|\right|$. Also

$$
\limsup _{n}\left\|y_{i, j \geq n}-y_{j}\right\|=\limsup _{n}\left|\left\|y_{i}-y_{j}\right\|\right|
$$

The above equality follows immediately from the following inequality, for $i, j \geq n$ :

$$
\left|\left\|y_{i}-y_{j}\right\|\right| \leq\left\|y_{i}-y_{j}\right\| \leq \frac{1}{\gamma_{n}}\left|\left\|y_{i}-y_{j}\right\|\right|
$$

This proves the lemma.

Now we will show that there exists a space with WNS such that with the $\Gamma$-norm it does not have WNS.

Lemma 2.7. Let $X$ be the space $c_{0}$ with the norm $\|x\|=\sup \left|b_{i}\right|+\sum_{i=1}^{\infty} \varepsilon_{i}\left|b_{i}\right|$, where $x=\sum_{i=1}^{\infty} b_{i} e_{i}$, $\varepsilon_{i}>0$ and $\sum_{i=1}^{\infty} \varepsilon_{i}<\infty$. Then $X$ has WNS.

Proof. Let $\left\{x_{n}\right\} \subset X$ be a weakly null nonzero sequence. We may assume that $x_{1} \neq 0$ and that there exists a block basic sequence $\left\{u_{n}\right\} \subset X$ with $\left\|u_{n}-x_{n}\right\| \underset{n \rightarrow \infty}{\rightarrow} 0$. Suppose that $u_{n}=\sum_{i=p_{n}}^{q_{n}} a_{i} e_{i}$ with $p_{n} \leq q_{n}<p_{n+1}$ for $n \in \mathbb{N}$ and that $x_{1}=\sum_{i=1}^{\infty} b_{i} e_{i}$. Let $k$ be such that $\sum_{i=1}^{k} \varepsilon_{i}\left|b_{i}\right|=\delta \neq 0$. Let $\varepsilon<\delta / 2, s>k$ with $\left\|Q_{s} x_{1}\right\|<\varepsilon$ and $n>s$. Then,

$$
\left\|x_{1}-u_{n}\right\|+\varepsilon \geq\left\|P_{s} x_{1}-u_{n}\right\|=\max \left(\left\|P_{s} x_{1}\right\|_{\infty},\left\|u_{n}\right\|_{\infty}\right)+\sum_{i=1}^{s} \varepsilon_{i}\left|b_{i}\right|+\sum_{i=p_{n}}^{q_{n}} \varepsilon_{i}\left|a_{i}\right| \geq\left\|u_{n}\right\|+\delta
$$

Hence, $\lim \sup _{n}\left\|x_{1}-x_{n}\right\| \geq \lim \sup _{n}\left\|x_{n}\right\|+\delta / 2$ and $\left\{x_{n}\right\}$ cannot be a diametral sequence, since for a diametral sequence $\left\{x_{n}\right\}$ it is true that $\lim _{n}\left\|x-x_{n}\right\|=\operatorname{diam}\left\{x_{n}\right\}$ for every $x \in$ $\overline{\operatorname{conv}}\left\{x_{n}\right\}$.

Lemma 2.8. Let $\Gamma=\left\{\gamma_{n}\right\} \subset(0,1)$ be an increasing sequence with $\lim _{n} \gamma_{n}=1$. Then there is a space $(X,\|\cdot\|)$ with WNS, such that $X$ with the $\Gamma$-norm $|\|\cdot\||$ does not have WNS.

Proof. Let $\left\{\gamma_{n_{j}}\right\}_{j}$ be a subsequence of $\left\{\gamma_{n}\right\}$ such that, if $\varepsilon_{n_{j}}=(1 / 3)\left(1 / \gamma_{n_{j}}-1\right)$, then $\sum_{i=1}^{\infty} \varepsilon_{n_{i}}<\infty$. Observe that $\varepsilon_{n_{j}}<\varepsilon_{n_{j+1}}$. Let $X$ be the space $c_{0}$ with the norm $\|x\|=\sup \left|a_{i}\right|+\sum_{i=1}^{\infty} \varepsilon_{n_{i}}\left|a_{n_{i}}\right|+$ $\sum_{i=1, i \neq n_{j}}^{\infty}\left(\left|a_{i}\right| / 2^{i}\right)$, where $x=\sum_{i=1}^{\infty} a_{i} e_{i}$. By Lemma 2.7, since $\sum_{i=1}^{\infty}\left(\varepsilon_{n_{i}}+1 / 2^{i}\right)<\infty$, we know that $X$ has WNS. Now let $|\|\cdot\|| \mid$ be the $\Gamma$-norm in $X$ with respect to $\left\{\gamma_{i}\right\}$. Let $u_{j}=e_{n_{j}}$; then $\gamma_{n_{j}}=1 /\left(1+3 \varepsilon_{n_{j}}\right)$, and thus

$$
\left|\left\|u_{j}\right\|\right|=\gamma_{n_{j}}\left(1+\varepsilon_{n_{j}}\right)<1
$$


and, if $j<m$,

$$
\begin{aligned}
\left|\left\|u_{j}-u_{m}\right\|\right| & =\max \left(\gamma_{n_{j}}\left(1+\varepsilon_{n_{j}}+\varepsilon_{n_{m}}\right), \gamma_{n_{m}}\left(1+\varepsilon_{n_{m}}\right)\right) \\
& \leq \max \left(\gamma_{n_{j}}\left(1+2 \varepsilon_{n_{j}}\right), \gamma_{n_{m}}\left(1+\varepsilon_{n_{m}}\right)\right)<1 .
\end{aligned}
$$

Therefore, since $0 \in \overline{\operatorname{conv}}\left\{u_{n}\right\}$ and $\lim _{n}\left|\left\|u_{n}\right\|\right|=1$, we have that $\operatorname{diam}_{\|\cdot\| \|}\left\{u_{n}\right\}=1$. Also, if $0 \leq \lambda_{i}, \sum_{i=1}^{n-1} \lambda_{i}=1$,

$$
||\left|u_{n}\left\||\leq||| \sum_{i=1}^{n-1} \lambda_{i} u_{i}-u_{n}\right\|\right| \leq 1
$$

Hence, $\left\{u_{n}\right\}$ is diametral in $(X,|\|\cdot\||)$.

The above example is another proof of the fact that for every $\varepsilon>0$ there are Banach spaces $X$ and $Y$ with $d(X, Y)<1+\varepsilon$ so that $X$ has WNS but $Y$ does not.

With regard to the coefficient $M W(X)$, we will see that, if $X$ has a premonotone basis and $\operatorname{MW}(X,\|\cdot\|)>1$, then $\operatorname{MW}(X,|\|\cdot\||)>1$ and we will show a sufficient condition for the reverse implication. For this we need the following lemma.

Lemma 2.9. Let $X$ be a Banach space with a basis. If

$$
\begin{gathered}
R W_{1}(a, X)=\sup \left\{\min \left(\lim \inf \left\|u_{n}+y\right\|, \lim \inf \left\|u_{n}-y\right\|\right): u_{n} \underset{w}{\longrightarrow} 0,\right. \\
\left\{u_{n}\right\} \subset B_{X} \text { is a block basic sequence, } \\
\|y\| \leq a \text { and support of } y \text { is finite }\},
\end{gathered}
$$

then $R W(a, X)=R W_{1}(a, X)$.

Proof. It is clear that $\mathrm{RW}_{1}(a, X) \leq \mathrm{RW}(a, X)$.

Now let $\varepsilon>0, x \in X,\|x\| \leq a$, and $\left\{x_{n}\right\} \subset B_{X}$, with $x_{n} \underset{w}{\rightarrow} 0$ such that $\min \left(\lim _{n} \| x_{n}+\right.$ $\left.x\left\|, \lim _{n}\right\| x_{n}-x \|\right)>\operatorname{RW}(a, X)-\varepsilon$. By passing to a subsequence, we may assume that there exist a block basic sequence $\left\{u_{n}\right\} \subset B_{\mathrm{X}}$ with $\left\|x_{n}-u_{n}\right\|<\varepsilon$ and $m \in \mathbb{N}$ such that $\left\|x-P_{m} x\right\|<\varepsilon$ and $\left\|P_{m} x\right\|<(1+\varepsilon)\|x\| \leq a(1+\varepsilon)$. Then,

$$
\begin{aligned}
& \left\|\frac{P_{m} x}{1+\varepsilon}+u_{n}\right\| \geq\left\|x_{n}+x\right\|-\left\|x_{n}-u_{n}\right\|-\left\|x-P_{m} x\right\|-\left\|P_{m} x\right\| \frac{\varepsilon}{1+\varepsilon}, \\
& \left\|\frac{P_{m} x}{1+\varepsilon}-u_{n}\right\| \geq\left\|x_{n}-x\right\|-\left\|x_{n}-u_{n}\right\|-\left\|x-P_{m} x\right\|-\left\|P_{m} x\right\| \frac{\varepsilon}{1+\varepsilon} .
\end{aligned}
$$

Let $y=P_{m} x /(1+\varepsilon)$; then $\|y\| \leq a$, and we conclude that $\mathrm{RW}_{1}(a, X) \geq \mathrm{RW}(a, X)-(3+a) \varepsilon$, thus proving the assertion. 
Similarly one can prove that, if $X$ is a space with a basis,

$$
\begin{aligned}
R(Y)=\sup \left\{\liminf _{n}\left\|u_{n}+x\right\|:\{x\},\left\{u_{n}\right\} \subset B_{Y}, u_{n} \underset{w}{\longrightarrow} 0,\right. \\
\left.\left\{u_{n}\right\} \text { is a block basic sequence and support of } x \text { is finite }\right\} .
\end{aligned}
$$

It is known (see $[6,8])$ that, if $X, Y$ are Banach spaces, then

$$
\operatorname{MW}(X) \leq \operatorname{MW}(Y) d(X, Y), \quad J(X) \leq J(Y) d(X, Y)
$$

So, if $X$ is a Banach space with a basis, $\Gamma=\left\{\gamma_{n}\right\}$ with $0 \leq \gamma_{n} \leq \gamma_{n+1} \leq 1$ and $\gamma_{1}>1 / \mathrm{MW}(X)$, and $Y$ is $X$ with the $\Gamma$-norm, then $\operatorname{MW}(Y) \geq \operatorname{MW}(X) / d(X, Y) \geq \gamma_{1} \operatorname{MW}(X)>1$, and if $\gamma_{1}>$ $1 / \operatorname{MW}(Y), \operatorname{MW}(X)>1$. Similarly, if $\gamma_{1}>J(X) / 2$, then $J(Y)<2$ and, if $\gamma_{1}>J(Y) / 2$, then $J(X)<2$. But the next proposition shows that in fact $\mathrm{MW}(X)>1$ always implies $\mathrm{MW}(Y)>1$. For the coefficient $J$, in general neither $J(X)<2$ implies $J(Y)<2$ nor the other way round, as we will see in Examples 2.16 and 2.17.

Proposition 2.10. Suppose that $X$ is a Banach space with a premonotone basis $\left\{e_{n}\right\}$. Then $M W=$ $M W(X,\|\cdot\|)>1$ implies that $M W_{1}=M W(X,|\|\cdot\||)>1$.

Proof. Let $\operatorname{RW}(a,(X,\|\cdot\|))=R(a)$ and $\operatorname{RW}(a,(X,\|\cdot\| \mid))=R_{1}(a)$. Suppose that $\mathrm{MW}_{1}=1$. Then, $R_{1}(a)=1+a$ for every $a>0$. Let $a>0$ and $0<\varepsilon<a, y \in X$ with finite support, $|\|y\|| \leq a$, and let $\left\{u_{n}\right\}$ be a weakly null block basic sequence with $\left|\left\|u_{n}\right\|\right| \leq 1$ such that $\lim _{n}\left|\left\|u_{n}+y\right\|\right|>1+a-\varepsilon$ and $\lim _{n}\left|\left\|u_{n}-y\right\|\right|>1+a-\varepsilon$. Then we may suppose that for every $n,\left|\left\|u_{n}+y\right\|\right|>1+a-\varepsilon$ and $\left|\left\|u_{n}-y\right\|\right|>1+a-\varepsilon$. Hence,

$$
\left|\left\|u_{n}\right\|\right| \geq 1-\varepsilon, \quad|\|y\|| \geq a-\varepsilon .
$$

We may also assume that the supports of $y$ and $u_{n}$ are disjoint. Let $u_{n}=\sum_{i=l_{n}}^{r_{n}} a_{i} e_{i}$ and $y=$ $\sum_{i=1}^{r} b_{i} e_{i}$.

Suppose that $\left|\left\|u_{n}+y\right\|\right|=\gamma_{m_{n}}\left\|\sum_{i=m_{n}}^{r_{n}}\left(a_{i}+b_{i}\right) e_{i}\right\|$ for some $m_{n} \leq r_{n}$. It is not possible that $m_{n}>r$, because this would mean that

$$
1+a-\varepsilon \leq\left|\left\|u_{n}+y\right\|\right|=\gamma_{m_{n}}\left\|\sum_{m_{n}}^{r_{n}} a_{i} e_{i}\right\| \leq\left|\left\|u_{n}\right\|\right| \leq 1
$$

So, by passing to a subsequence if necessary, we may assume that for every $n$ we have that $m_{n}=i_{0} \leq r$. Thus,

$$
\begin{aligned}
1+a-\varepsilon & \leq\left|\left\|u_{n}+y\right\|\right|=\gamma_{i_{0}}\left\|\sum_{i=i_{0}}^{r_{n}}\left(a_{i}+b_{i}\right) e_{i}\right\| \\
& \leq \gamma_{i_{0}}\left(\left\|\sum_{i=l_{n}}^{r_{n}} a_{i} e_{i}\right\|+\left\|\sum_{i=i_{0}}^{r} b_{i} e_{i}\right\|\right) \leq \frac{\gamma_{i_{0}}}{\gamma_{l_{n}}}+a .
\end{aligned}
$$


Since $\lim _{n} \gamma_{n}=1$, by passing to the limit, we obtain that

$$
\gamma_{i_{0}} \geq 1-\varepsilon
$$

Similarly, there exists $\gamma_{i_{1}} \geq 1-\varepsilon$ with

$$
1+a-\varepsilon \leq\left|\left\|u_{n}-y\right\|\right| \leq \frac{\gamma_{i_{1}}}{\gamma_{l_{n}}}+a
$$

Suppose that $i_{1} \geq i_{0}$, and let $y_{0}=\sum_{i=i_{0}}^{r} b_{i} e_{i}$ and $y_{1}=\sum_{i=i_{1}}^{r} b_{i} e_{i}$. Then, since the basis is premonotone, $1+a-\varepsilon \leq\left|\left\|u_{n}+y\right\|\right|=\left|\left\|u_{n}+y_{0}\right\|\right| \leq\left\|u_{n}+y_{0}\right\|$ and $1+a-\varepsilon \leq\left|\left\|u_{n}-y\right\|\right|=$ $\left|\left\|u_{n}-y_{1}\right\|\right| \leq\left\|u_{n}-y_{1}\right\| \leq\left\|u_{n}-y_{0}\right\| ;$ thus,

$$
a-\varepsilon \leq\left|\left\|y_{0}\right\|\right| \leq|\|y\|| \leq a .
$$

Therefore,

$$
a-\varepsilon \leq\left|\left\|y_{0}\right\|\right| \leq\left\|y_{0}\right\| \leq \frac{1}{\gamma_{i_{0}}}\left|\left\|y_{0}\right\|\right| \leq \frac{a}{\gamma_{i_{0}}} \leq \frac{a}{1-\varepsilon}
$$

and $\left|\left\|y_{0}\right\|-a\right| \leq \max \{\varepsilon, a \varepsilon /(1-\varepsilon)\}$. Further, since $\gamma_{i_{0}} \leq \gamma_{n_{n}}$,

$$
1-\varepsilon \leq\left|\left\|u_{n}\right\|\right| \leq\left\|u_{n}\right\| \leq \frac{1}{\gamma_{n}}\left\|u_{n}\right\| \mid \leq \frac{1}{\gamma_{n}} \leq \frac{1}{1-\varepsilon}
$$

and $\left|\left\|u_{n}\right\|-1\right| \leq \varepsilon /(1-\varepsilon)$.

Hence,

$$
\begin{aligned}
\left\|\frac{u_{n}}{\left\|u_{n}\right\|}+\frac{y_{0}}{\left\|y_{0}\right\|} a\right\| & \geq\left\|u_{n}+y_{0}\right\|-\left|\left\|u_{n}\right\|-1\right|-\left|\left\|y_{0}\right\|-a\right| \\
& \geq 1+a-\varepsilon-\frac{\varepsilon}{1-\varepsilon}-\max \left\{\varepsilon, \frac{a \varepsilon}{1-\varepsilon}\right\} .
\end{aligned}
$$

Similarly,

$$
\left\|\frac{u_{n}}{\left\|u_{n}\right\|}-\frac{y_{0}}{\left\|y_{0}\right\|} a\right\| \geq 1+a-\varepsilon-\frac{\varepsilon}{1-\varepsilon}-\max \left\{\varepsilon, \frac{a \varepsilon}{1-\varepsilon}\right\}
$$

We deduce that $\min \left(\lim \inf _{n}\left\|\left(u_{n} /\left\|u_{n}\right\|\right)+\left(y_{0} /\left\|y_{0}\right\|\right) a\right\|, \liminf _{n}\left\|\left(u_{n} /\left\|u_{n}\right\|\right)-\left(y_{0} /\left\|y_{0}\right\|\right) a\right\|\right) \geq$ $1+a-\varepsilon-\varepsilon /(1-\varepsilon)-\max \{\varepsilon, a \varepsilon /(1-\varepsilon)\}$, and letting $\varepsilon$ tend to zero we obtain $R(a)=1+a$ and $\mathrm{MW}=1$.

Examples 2.14 and 2.17 exhibit spaces in which $\operatorname{MW}(X,\|\cdot\|)=1$ and $\operatorname{MW}(X,|\|\cdot\||)>1$. There is however a special case in which $\operatorname{MW}(X,\|\cdot\|) \geq \operatorname{MW}(X, \mid\|\cdot\| \|)$. 
Recall that a basis $\left\{e_{n}\right\}$ of a Banach space $X$ is 1-spreading if, whenever $x=\sum_{i=1}^{\infty} a_{i} e_{i} \in$ $X$ and $\left\{e_{n_{i}}\right\}_{i}$ is a subsequence of $\left\{e_{n}\right\}, \sum_{i=1}^{\infty} a_{i} e_{n_{i}} \in X$ and $\left\|\sum_{i=1}^{\infty} a_{i} e_{i}\right\|=\left\|\sum_{i=1}^{\infty} a_{i} e_{n_{i}}\right\|$.

Proposition 2.11. If $X$ is a Banach space with a premonotone 1-spreading basis, then $M W(X,\|\cdot\|) \geq$ $M W(X,|\|\cdot\||)$.

Proof. Let $T_{m}: X \rightarrow X$ be the translation given by $T_{m} \sum_{i=1}^{\infty} a_{i} e_{i}=\sum_{i=1}^{\infty} a_{i} e_{i+m}$, and let $a>$ $0, y \in X,\|y\| \leq a,\left\{u_{n}\right\} \subset B_{X}$, where $y$ has finite support and $\left\{u_{n}\right\}$ is a weakly null block basic sequence such that $\min \left(\left\|y+u_{n}\right\|,\left\|y-u_{n}\right\|\right)>R W(a,(X,\|\cdot\|))-\varepsilon$ for every $n$. Let $m \in \mathbb{N}$; then there exists $N \in \mathbb{N}$ such that for $n>N$ the supports of $T_{m} y$ and $u_{n}$ are disjoint, and thus, since the basis is 1-spreading, $\left\|y-u_{n}\right\|=\left\|T_{m} y-u_{n}\right\|$ and $\left\|y+u_{n}\right\|=\left\|T_{m} y+u_{n}\right\|$. Then, $\left|\left\|T_{m} y\right\|\right| \leq\left\|T_{m} y\right\| \leq a,\left\|u_{n}\right\| \mid \leq\left\|u_{n}\right\| \leq 1$ and for $n>N$

$$
\begin{aligned}
& \left|\left\|T_{m} y-u_{n}\right\|\right| \geq \gamma_{m}\left\|T_{m} y-u_{n}\right\|=\gamma_{m}\left\|y-u_{n}\right\|, \\
& \left|\left\|T_{m} y+u_{n}\right\|\right| \geq \gamma_{m}\left\|T_{m} y+u_{n}\right\|=\gamma_{m}\left\|y+u_{n}\right\| .
\end{aligned}
$$

Hence, $\operatorname{RW}(a,(X,|\|\cdot\||)) \geq \gamma_{m} \operatorname{RW}(a,(X,\|\cdot\|))$, and, by passing to the limit as $m$ tends to infinity, we get $\operatorname{RW}(a,(X,|\|\cdot\||)) \geq \operatorname{RW}(a,(X,\|\cdot\|))$ and thus the desired result.

Similarly to Propositions 2.10 and 2.11 we can prove the following.

Proposition 2.12. Suppose that $X$ is a Banach space with a premonotone basis $\left\{e_{n}\right\}$. Then $R=$ $R(X,\|\cdot\|)<2$ implies that $R_{1}=R(X,\|\cdot\| \|)<2$ and, if the basis is premonotone and 1-spreading, then $R=R(X,\|\cdot\|) \leq R(X,\|\cdot\| \mid)=R_{1}$.

Corollary 2.13. If $X$ is a Banach space with a premonotone 1-spreading basis, then $M W_{1}>1$ if and only if $M W>1$; also $R_{1}<2$ if and only if $R<2$.

Next we will show an example of a space without a 1-spreading basis, such that $R=2$, $\mathrm{MW}=1$ but $R_{1}<2$ and thus $\mathrm{MW}_{1}>1$.

Example 2.14. Let $X$ be $c_{0}$ with the following norm:

$$
\left\|\left\{a_{n}\right\}\right\|=\left|a_{1}\right|+\max _{i \geq 2}\left|a_{i}\right|
$$

Let $\left\{e_{n}\right\}$ denote the canonical basis of $c_{0}$. Then for every $a>0,\left\|a e_{1}+e_{n}\right\|=\left\|a e_{1}-e_{n}\right\|=1+a$, and thus $R=2$ and MW $=1$. On the other hand let $x \in X$ with finite support and \|\|$x \| \mid \leq 1$ and suppose that $\left\{u_{n}\right\}$ is a block basic sequence with $\left\|u_{n}\right\| \mid \leq 1$ for $n \in N, u_{n}=\sum_{i=m_{n}}^{r_{n}} a_{i} e_{i}$ with $m_{n} \leq r_{n}<m_{n+1}$ and the support of $x$ does not intersect the support of $u_{n}$. Then, for every $m \geq 2$ and $n \geq m, \gamma_{m}\left\|Q_{m} x+u_{n}\right\|=\max \left(\gamma_{m}\left\|Q_{m} x\right\|_{c_{0}}, \gamma_{m}\left\|u_{n}\right\|_{c_{0}}\right) \leq 1$,

$$
\gamma_{1}\left\|x+u_{n}\right\| \leq 1+\gamma_{1}\left\|u_{n}\right\|_{c_{0}} \leq 1+\frac{\gamma_{1}}{\gamma_{m_{n}}} .
$$

Thus, $\lim \inf \left|\left\|x+u_{n}\right\|\right| \leq 1+\gamma_{1}$. Hence $R_{1} \leq 1+\gamma_{1}<2$ and, by (2.9), $\mathrm{MW}_{1}>1$.

With regard to the coefficient $J$ we have the following which is proved similarly to Proposition 2.11. 
Proposition 2.15. Suppose that $X$ is a Banach space with a premonotone 1-spreading basis $\left\{e_{n}\right\}$. Then $J(X,\|\cdot\|) \leq J(X,\|\cdot\| \mid)$.

In general neither $J(X,\|\cdot\|)<2$ implies $J(X,|\|\cdot\||)<2$ nor the other way round, as the following examples show.

Example 2.16. Let $1>\mu \geq 1 / \sqrt{2}$ and $X=\mathbb{R}_{\mu}^{2} \oplus_{2} l_{2}$, where $\mathbb{R}_{\mu}^{2}=\left(\mathbb{R}^{2},\|\cdot\|_{(\mu)}\right)$ and $\left\|\left(x_{1}, x_{2}\right)\right\|_{(\mu)}=$ $\max \left(\left|x_{1}\right|,\left|x_{2}\right|, \mu\left(\left|x_{1}\right|+\left|x_{2}\right|\right)\right)$. Then, if $\mu \leq \gamma_{2} /\left(\gamma_{1}+\gamma_{2}\right), J(X)<2$ but for every $\Gamma, J(X,|||\cdot \||)=2$.

Since $\mu \geq 1 / \sqrt{2}$, it is easy to see that for $x=\left(x_{1}, x_{2}\right) \in \mathbb{R}^{2}$,

$$
\frac{\mu}{\sqrt{1-2 \mu+2 \mu^{2}}}\|x\|_{2} \leq\|x\|_{(\mu)} \leq \mu \sqrt{2}\|x\|_{2} .
$$

Thus, $d\left(l_{2}, X\right) \leq d\left(\mathbb{R}^{2}, \mathbb{R}_{\mu}^{2}\right)=\sqrt{2} \sqrt{1-2 \mu+2 \mu^{2}}<\sqrt{2}$, and by (2.22), since $J\left(l_{2}\right)=\sqrt{2}$, we obtain that $J(X)<2$.

Now let $\Gamma=\left\{\gamma_{n}\right\}, \mu \leq \gamma_{2} /\left(\gamma_{1}+\gamma_{2}\right), x=\left(1 / \gamma_{1}, 1 / \gamma_{2}, 0,0, \ldots\right)$, and $y=$ $\left(-1 / \gamma_{1}, 1 / \gamma_{2}, 0,0, \ldots\right)$. Then $|\|x\||=|\|y\||=1$ but $|\|x+y\||=|\|x-y\||=2$.

This last example is another proof of the known fact that for every $\varepsilon>0$ there are Banach spaces $X$ and $Y$ with $d(X, Y)<1+\varepsilon, J(X)<2$ but $J(Y)=2$. In the following example we exhibit a space $X$ with $J(X)=2$ such that $J(X,\|\cdot\| \mid)<2$.

Example 2.17. Let $X=\left(l_{2},\|\cdot\|\right)$, where, for $x=\left(a_{n}\right) \in l_{2},\|x\|=\left|a_{1}\right|+\left(\sum_{i=2}^{\infty} a_{i}^{2}\right)^{1 / 2}$. Then $J(X)=2, \operatorname{MW}(X)=1$, and, if $\Gamma$ is such that $\gamma_{2}>1 / \sqrt{2}$ and $\gamma_{1} / \gamma_{2}<1 / \sqrt{2}, J(X,|\|\cdot\||)<2$ and thus $\operatorname{MW}(X,|\|\cdot\||)>1$.

Obviously if $\left\{e_{n}\right\}$ is the canonical basis of $l_{2}$, and $a>0$, then $\left\|a e_{1}+e_{n}\right\|=\left\|a e_{1}-e_{n}\right\|=$ $1+a$ for $n>1$ and thus $J(X)=2$ and $\operatorname{MW}(X)=1$.

Suppose now that $J(Y)=J(X,\|\| \cdot \| \mid)=2$. Then there exist sequences $\left\{x_{n}\right\},\left\{y_{n}\right\} \subset B_{Y}$ such that $\lim _{n}\left|\left\|x_{n}+y_{n}\right\|\right|=\lim _{n}\left|\left\|x_{n}-y_{n}\right\|\right|=2$ and $\left\{m_{n}\right\},\left\{l_{n}\right\} \subset \mathbb{N}$ so that

$$
\begin{gathered}
\left|\left\|x_{n}+y_{n}\right\|\right|=\gamma_{m_{n}}\left\|Q_{m_{n}}\left(x_{n}+y_{n}\right)\right\|, \\
\left|\left\|x_{n}-y_{n}\right\|\right|=\gamma_{n}\left\|Q_{l_{n}}\left(x_{n}-y_{n}\right)\right\| .
\end{gathered}
$$

By passing to a subsequence, we may assume that, for every $n \in \mathbb{N}, e_{1}^{*}\left(x_{n}\right) \geq 0, e_{1}^{*}\left(y_{n}\right) \geq$ 0 and $e_{1}^{*}\left(x_{n}\right) \geq e_{1}^{*}\left(y_{n}\right)$, and that the subsequence satisfies one of the next three cases:

(1) $m_{n}>1$ and $l_{n}>1$ for every $n$,

(2) $m_{n}=l_{n}=1$ for every $n$,

(3) $m_{n}=1$ and $l_{n}>1$ for every $n$.

Observe that

$$
\gamma_{m}\left\|Q_{m}(x+y)\right\| \geq 2-\varepsilon \text { implies } \gamma_{m}\left\|Q_{m}(x)\right\| \geq 1-\varepsilon \text { and } \gamma_{m}\left\|Q_{m}(y)\right\| \geq 1-\varepsilon \text {. }
$$


Case 1. Let $Z=\left(l_{2},|\|\cdot\||_{\Gamma_{1}}\right)$, where $\left|\left\|\cdot|\||_{\Gamma_{1}}\right.\right.$ is the $\Gamma_{1}=\left\{\gamma_{i}\right\}_{i=2}^{\infty}$-norm. Then

$$
\begin{array}{cl}
\left|\left\|Q_{2} x_{n}\right\|\right|_{\Gamma_{1}} \leq 1, \quad & \left|\left\|Q_{2} y_{n}\right\|\right|_{\Gamma_{1}} \leq 1, \\
\left|\left\|Q_{2}\left(x_{n}+y_{n}\right)\right\|\right|_{\Gamma_{1}} \geq 2-\varepsilon, \quad & \left|\left\|Q_{2}\left(x_{n}-y_{n}\right)\right\|\right|_{\Gamma_{1}} \geq 2-\varepsilon .
\end{array}
$$

Since $1 / \gamma_{2}<\sqrt{2}$, then, by $(2.2), d\left(Z, l_{2}\right)<\sqrt{2}$ and, by $(2.22)$, since $J\left(l_{2}\right)=\sqrt{2,} J(Z)<2$ and this is a contradiction.

Case 2. Let $\varepsilon>0$. Suppose that $x=\sum_{i=1}^{\infty} a_{i} e_{i}, y=\sum_{i=1}^{\infty} b_{i} e_{i} \in B_{Y}, a_{1}>0, b_{1}>0, a_{1}>b_{1}$ and

$$
\begin{aligned}
& \gamma_{1}\left(\sum_{i=2}^{\infty}\left(a_{i}+b_{i}\right)^{2}\right)^{1 / 2} \geq 2-\varepsilon-\gamma_{1}\left(a_{1}+b_{1}\right), \\
& \gamma_{1}\left(\sum_{i=2}^{\infty}\left(a_{i}-b_{i}\right)^{2}\right)^{1 / 2} \geq 2-\varepsilon-\gamma_{1}\left(a_{1}-b_{1}\right) .
\end{aligned}
$$

Squaring both inequalities and adding them, since $\gamma_{2}\left(\sum_{i=2}^{\infty} a_{i}^{2}\right)^{1 / 2} \leq|\|x\||$, we get

$$
\frac{2 \gamma_{1}^{2}}{r_{2}^{2}} \geq \gamma_{1}^{2} \sum_{i=2}^{\infty}\left(a_{i}^{2}+b_{i}^{2}\right) \geq\left(2-\varepsilon-\gamma_{1} a_{1}\right)^{2}+\gamma_{1}^{2} b_{1}^{2} \geq\left(2-\varepsilon-\gamma_{1} a_{1}\right)^{2}
$$

By passing to the limit as $\varepsilon \rightarrow 0$, since $\gamma_{1} a_{1} \leq 1$, we obtain that $\sqrt{2}\left(\gamma_{1} / \gamma_{2}\right) \geq 2-\gamma_{1} a_{1} \geq 1$, and this contradicts $\gamma_{1} / \gamma_{2}<1 / \sqrt{2}$.

Case 3. Let $2>\varepsilon>0$. Suppose that $x=\sum_{i=1}^{\infty} a_{i} e_{i}, y=\sum_{i=1}^{\infty} b_{i} e_{i} \in B_{Y}, a_{1}>0, b_{1} \geq 0, a_{1}>b_{1}$ and $|\|x+y\||=\gamma_{1}\left(\left|a_{1}+b_{1}\right|+\left(\sum_{i=2}^{\infty}\left(a_{i}+b_{i}\right)^{2}\right)^{1 / 2}\right) \geq 2-\varepsilon$ and $\|x-y\| \mid=\gamma_{m}\left(\sum_{i=m}^{\infty}\left(a_{i}-b_{i}\right)^{2}\right)^{1 / 2} \geq$ $2-\varepsilon$. Then, by (2.38),

$$
\gamma_{m}\left(\sum_{i=m}^{\infty} a_{i}^{2}\right)^{1 / 2} \geq 1-\varepsilon
$$


International Journal of Mathematics and Mathematical Sciences

Since in $l_{2}$ if $u, v \in B_{l_{2}}$, one has that $\|u-v\| \geq \delta$ implies $\|u+v\| \leq 2 \sqrt{1-(\delta / 2)^{2}}$, then $\gamma_{m}\left(\sum_{i=m}^{\infty}\left(a_{i}+b_{i}\right)^{2}\right)^{1 / 2} \leq \sqrt{4 \varepsilon-\varepsilon^{2}}$. Also

$$
\begin{aligned}
2-\varepsilon & \leq \gamma_{1}\left(\left|a_{1}+b_{1}\right|+\left(\sum_{i=2}^{m-1}\left(a_{i}+b_{i}\right)^{2}\right)^{1 / 2}+\left(\sum_{i=m}^{\infty}\left(a_{i}+b_{i}\right)^{2}\right)^{1 / 2}\right) \\
& \leq \gamma_{1}\left|a_{1}+b_{1}\right|+\gamma_{1}\left(\sum_{i=2}^{m-1}\left(a_{i}+b_{i}\right)^{2}\right)^{1 / 2}+\frac{\gamma_{1}}{\gamma_{m}} \sqrt{4 \varepsilon-\varepsilon^{2}} \\
& \leq \gamma_{1}\left|a_{1}+b_{1}\right|+\gamma_{1}\left(\sum_{i=2}^{m-1}\left(a_{i}+b_{i}\right)^{2}\right)^{1 / 2}+\sqrt{4 \varepsilon-\varepsilon^{2}} .
\end{aligned}
$$

Let $\phi=\varepsilon+\sqrt{4 \varepsilon-\varepsilon^{2}}$; then

$$
2-\phi \leq \gamma_{1}\left|a_{1}+b_{1}\right|+\gamma_{1}\left(\sum_{i=2}^{m-1}\left(a_{i}+b_{i}\right)^{2}\right)^{1 / 2} .
$$

By (2.38), $\gamma_{1}\left|a_{1}\right|+\gamma_{1}\left(\sum_{i=2}^{m-1} a_{i}^{2}\right)^{1 / 2} \geq 1-\phi$, and since, for $A, B>0$,

$$
(A+B)^{1 / 2}-A^{1 / 2}=\frac{B}{(A+B)^{1 / 2}+A^{1 / 2}}
$$

we have, using (2.42), that

$$
\begin{aligned}
& \geq \gamma_{1}\left|a_{1}\right|+\gamma_{1}\left(\sum_{i=2}^{\infty} a_{i}^{2}\right)^{1 / 2} \\
& \geq \gamma_{1}\left|a_{1}\right|+\gamma_{1}\left(\sum_{i=2}^{m-1} a_{i}^{2}+\frac{(1-\varepsilon)^{2}}{r_{m}^{2}}\right)^{1 / 2} \\
& =\gamma_{1}\left|a_{1}\right|+\gamma_{1}\left(\sum_{i=2}^{m-1} a_{i}^{2}\right)^{1 / 2}+\frac{\gamma_{1}(1-\varepsilon)^{2}}{r_{m}^{2}} \frac{1}{(A+B)^{1 / 2}+A^{1 / 2}} \\
& \geq 1-\phi+\frac{\gamma_{1}(1-\varepsilon)^{2}}{\gamma_{m}^{2}} \frac{1}{(A+B)^{1 / 2}+A^{1 / 2}},
\end{aligned}
$$

where $A=\left(\sum_{i=2}^{m-1} a_{i}^{2}\right)^{1 / 2}$ and $B=(1-\varepsilon)^{2} / \gamma_{m}^{2}$. But

$$
(A+B)^{1 / 2}+A^{1 / 2} \leq\left(\frac{1}{\gamma_{2}^{2}}+\frac{(1-\varepsilon)^{2}}{\gamma_{m}^{2}}\right)^{1 / 2}+\frac{1}{\gamma_{2}} \leq \frac{3}{\gamma_{2}} ;
$$


therefore,

$$
r_{1}<\frac{\gamma_{1}}{r_{m}^{2}} \leq \frac{\phi}{(1-\varepsilon)^{2}} \frac{3}{\gamma_{2}}
$$

and, taking the limit as $\varepsilon \rightarrow 0$, we get $\gamma_{1}=0$ which is a contradiction.

Hence, $J(X,|\|\cdot\||)<2$, and, by $(2.10)$, we have that $\mathrm{MW}(X,|\|\cdot\||)>1$.

\section{Acknowledgments}

The authors thank the referees for their detailed review and valuable observations. This work was partially funded by Grant SEP CONACYT 102380.

\section{References}

[1] P. N. Dowling, C. J. Lennard, and B. Turett, "Renormings of $l_{1}$ and $c_{0}$ and fixed point properties," in Handbook of Metric Fixed Point Theory, pp. 269-297, Kluwer Academic Publishers, Dordrecht, The Netherlands, 2001.

[2] P.-K. Lin, "There is an equivalent norm on $l_{1}$ that has the fixed point property," Nonlinear Analysis: Theory, Methods \& Applications, vol. 68, no. 8, pp. 2303-2308, 2008.

[3] A. Jiménez-Melado, "Stability of weak normal structure in James quasi reflexive space," Bulletin of the Australian Mathematical Society, vol. 46, no. 3, pp. 367-372, 1992.

[4] H. Fetter and B. Gamboa de Buen, "Geometric properties related to the fixed point property in Banach spaces," Revista de la Real Academia de Ciencias Exactas, Físicas y Naturales, vol. 94, no. 4, pp. 431-436, 2000.

[5] J. García Falset, "The fixed point property in Banach spaces with the NUS-property," Journal of Mathematical Analysis and Applications, vol. 215, no. 2, pp. 532-542, 1997.

[6] J. García Falset, E. Llorens-Fuster, and E. M. Mazcuñán-Navarro, "Uniformly nonsquare Banach spaces have the fixed point property for nonexpansive mappings," Journal of Functional Analysis, vol. 233, no. 2, pp. 494-514, 2006.

[7] H. Fetter and B. Gamboa de Buen, "Banach spaces with a basis that are hereditarily asymptotically isometric to $l_{1}$ and the fixed point property," Nonlinear Analysis: Theory, Methods \& Applications, vol. 71, no. 10 , pp. 4598-4608, 2009.

[8] M. Kato, L. Maligranda, and Y. Takahashi, "On James and Jordan-von Neumann constants and the normal structure coefficient of Banach spaces," Studia Mathematica, vol. 144, no. 3, pp. 275-295, 2001. 


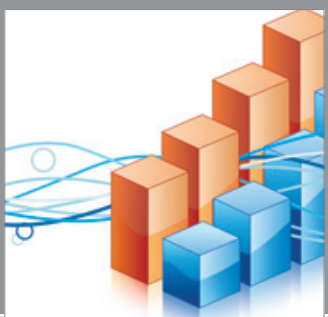

Advances in

Operations Research

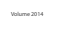

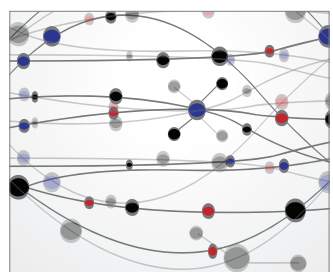

\section{The Scientific} World Journal
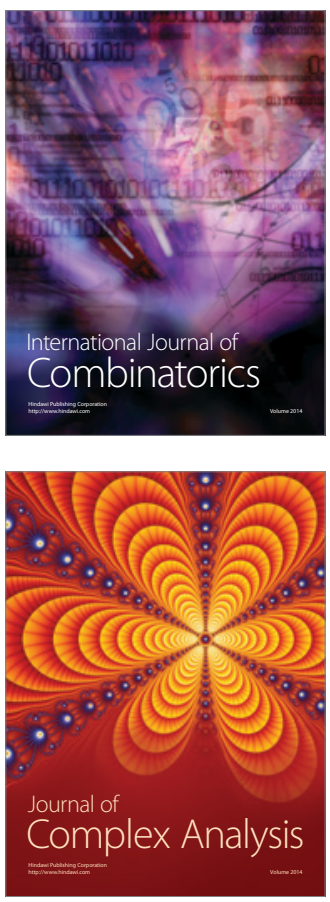

International Journal of

Mathematics and

Mathematical

Sciences
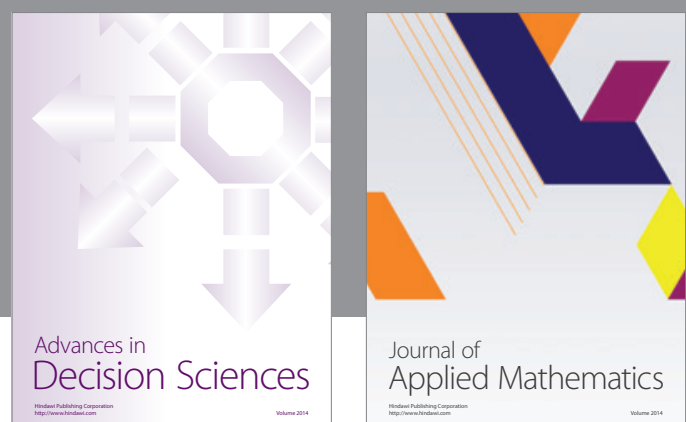

Journal of

Applied Mathematics
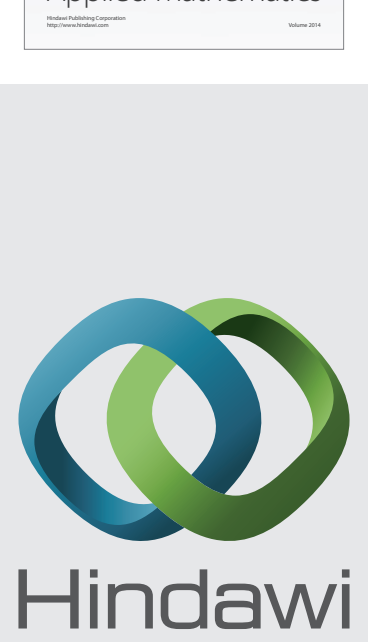

Submit your manuscripts at http://www.hindawi.com
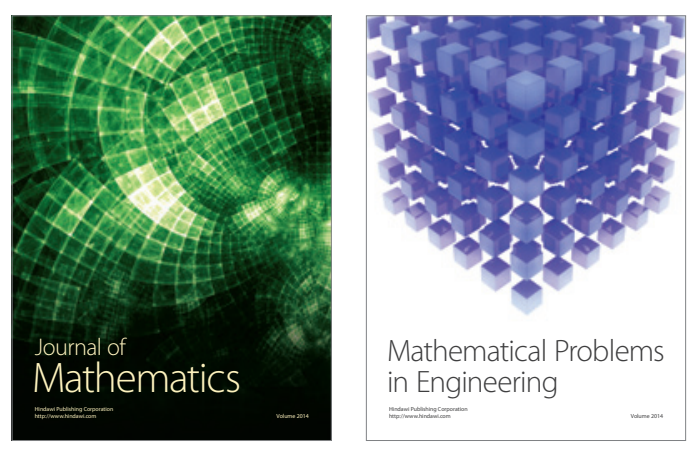

Mathematical Problems in Engineering
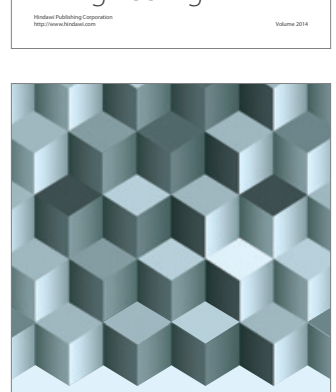

Journal of

Function Spaces
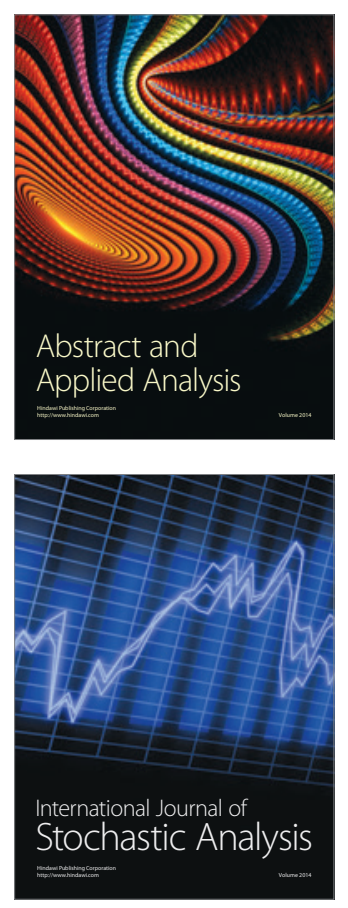

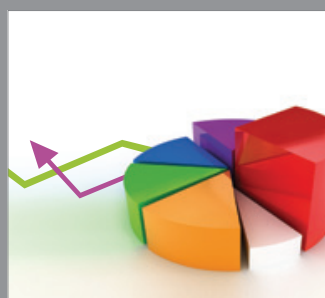

ournal of

Probability and Statistics

Promensencen
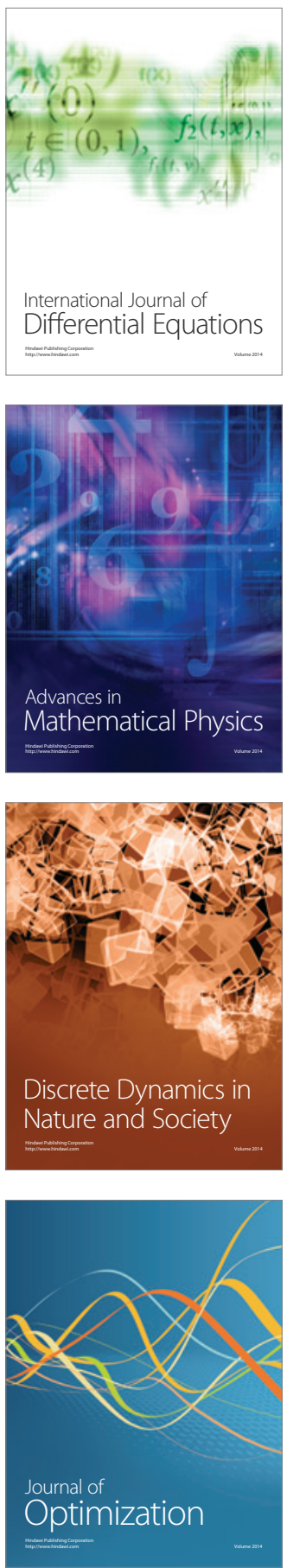